\title{
La protection des données personnelles dans le domaine des relations de travail en Espagne
}

Jesús Cruz Villalón

\section{(2) OpenEdition}

1 Journals

\section{Édition électronique}

URL : https://journals.openedition.org/rdctss/1218

DOI : $10.4000 /$ rdctss. 1218

ISSN : 2262-9815

Éditeur

Centre de droit comparé du travail et de la sécurité sociale

\section{Édition imprimée}

Date de publication : 1 avril 2020

Pagination : 114-127

ISSN : 2117-4350

\section{Référence électronique}

Jesús Cruz Villalón, «La protection des données personnelles dans le domaine des relations de travail en Espagne ", Revue de droit comparé du travail et de la sécurité sociale [En ligne], 1 | 2020, mis en ligne le 01 novembre 2021, consulté le 28 juin 2022. URL : http://journals.openedition.org/rdctss/1218 ; DOI : https://doi.org/10.4000/rdctss. 1218

\section{cc)}

La Revue de droit comparé du travail et de la sécurité sociale est mise à disposition selon les termes de la Licence Creative Commons Attribution - Pas d'Utilisation Commerciale - Pas de Modification 4.0 International. 


\section{LA PROTECTION DES DONNÉES PERSONNELLES DANS LE DOMAINE DES RELATIONS DE TRAVAIL EN ESPAGNE*}

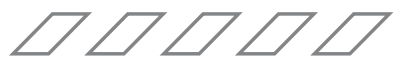

\section{RÉSUMÉ}

Cette étude vise à analyser l'impact de la reconnaissance du droit à la protection des données personnelles sur les relations de travail, à la lumière des législations européenne et espagnole. Elle tente plus particulièrement d'établir une distinction entre le droit à l'intimité et le droit à la vie privée. De même, elle tente de mettre en évidence les principaux défis et les particularités liés à la protection de la sphère privée du travailleur : son éventuelle extension au travail indépendant, les limites indirectes découlant de la reconnaissance du droit à la protection des données personnelles de l'employeur, les limites du consentement dans le domaine du travail et les facteurs conditionnant l'exercice du pouvoir de contrôle de l'employeur.

MOTS CLÉS : Droits fondamentaux du travail, intimité et vie privée, limites des entreprises.

\section{ABSTRACT}

This study aims to analyze the impact on industrial relations of the recognition of the right to personal data protection, in the light of European and Spanish legislation. It makes especially an attempt to mark the differences between the right to privacy and the most restricted of the intimate sphere. Likewise, it tries to highlight the main challenges and singularities of the protection of the sphere of the private with respect to the worker: it possible extension to self-employed worker, the indirect limitations derived from the recognition of the right to the protection of personal data of the employer, the limited space of labour consent and the requirement in this matter of employer control powers.

KEYWORDS : Fundamental Labour Rights, Personal and Family Privacy, Business Limits.

1 Ce travail a été réalisé dans le cadre du projet de recherche « Les droits fondamentaux face aux transformations du travail dans la nouvelle économie », DER2017-83488-C4-1-R, du Ministère de l'économie, de l'industrie et de la compétitivité. 
a protection de la vie privée, comme point de départ de la prise en compte de la protection des données personnelles, prend racine dans la Constitution Espagnole de 1978 (ci-après CE), qui intègre à la relation des droits fondamentaux et des libertés publiques la reconnaissance du droit à l'honneur, à l'intimité personnelle et à l'image (art. 18.1 CE). Cette disposition fera prochainement l'objet d'un nouveau règlement par le biais d'une loi organique, indispensable pour mettre en œuvre les droits fondamentaux susmentionnés dans notre système juridique (art. 81.1 CE) ${ }^{1}$.

Cependant, l'évolution de la législation visait au départ essentiellement les relations juridiques privées en dehors de la sphère du travail. S'agissant du domaine du travail, les dispositions y relatives se limitaient, dans le texte initial du Statut des travailleurs de 1980, à des rappels génériques à la vie privée en tant que droit fondamental des travailleurs (art. 4.e) et à certaines mentions visant dans un premier temps à limiter l'exercice du pouvoir de contrôle de l'employeur en cas de fouilles des effets personnels du travailleur (art. 18), puis étendues dans un second temps à limiter la transmission de certaines informations contractuelles aux représentants de salariés (art. 8.4).

Rédigée lors de la phase transitoire qui a suivi l'adoption des Constitutions européennes après la Seconde Guerre mondiale, la Constitution Espagnole est novatrice dans le sens où elle confère un mandat express au législateur afin de limiter, en cas de nécessité, l'utilisation de l'informatique pour garantir l'honneur et l'intimité personnelle et familiale des citoyens, ainsi que le plein exercice de leurs droits (article 18.4). A la lumière de ce qui précède, ceci a conduit la Cour constitutionnelle à considérer que la protection des données personnelles doit être reconnue comme un droit de valeur constitutionnelle, au même titre que les libertés publiques et fondamentales. La Cour estime donc, depuis un certain temps, que ce droit a acquis sa propre autonomie constitutionnelle et dépasse le seul cadre du droit à l'intimité, qui se traduit par un droit de contrôle sur les données relatives à la personne elle-même².

Ce même raisonnement a conduit la Cour constitutionnelle à limiter, dans le domaine spécifique du travail, l'utilisation de certaines informations détenues par l'employeur. C'est le cas notamment des données relatives à la syndicalisation des travailleurs, utilisées par l'employeur pour présupposer - sans preuve valable - que les membres d'un syndicat

1 Loi organique 1/1982 du 5 mai 1982 relative à la protection civile du droit à l'honneur, à l'intimité personnelle et familiale et à l'image, Bulletin Officiel de l'Etat (ci-après BOE) du 14 mai.

2 SSTC 143/1994 du 9 mai 1994 (BOE 13 juin 1994); 292/2000 du 30 novembre (BOE 4 janvier 2001) ; 76/2019 du 22 mai (BOE 25 juin 2019). 
appelant à la grève y ont participé et déduire ensuite de leur salaire les jours de grève ${ }^{3}$. Ce droit fondamental a été instauré par une loi à la fin du siècle dernier,

assortie de réformes successives de plus en plus poussées ${ }^{4}$. Toutefois, ces réglementations sont essentiellement de nature transversale, touchant tous les types d'actes juridiques et de relations sociales, notamment dans le domaine du travail, bien que les particularités des relations de travail soient peu prises en compte. Seul le dernier règlement, adopté fin 2018 , fait pour la première fois spécifiquement référence à la protection des données à caractère personnel dans le domaine du travail.

Au fil du temps, I'Union Européenne interviendra avec davantage de force et d'intensité, en adoptant des réglementations bien connues qui accordent une grande attention au droit à la protection des données, et qui auront une influence décisive sur la réglementation nationale. La loi espagnole de 1999 se basait déjà sur la transposition de la directive de $1995^{5}$, depuis lors abrogée. À l'heure actuelle, le règlement de l'UE ${ }^{6}$ et la directive de $2016^{7}$ constituent les deux textes essentiels. La réglementation espagnole en vigueur fin $2018 \mathrm{se}$ présente comme un complément et une transposition des précédentes réglementations européennes. Rappelons à nouveau que ces règlements sont de nature transversale pour toutes les relations juridiques quel que soit le domaine.

Enfin, il faut mentionner la reconnaissance, dans la Charte des droits fondamentaux de I'Union Européenne, du droit de toute personne à la protection de ses données à caractère personnel, dès lors que ces données sont traitées de manière loyale, à des fins spécifiques et sur la base du consentement de la personne concernée (ou en vertu d'un autre fondement légitime prévu par la loi), avec une référence expresse au droit de toute personne d'avoir accès aux données collectées la concernant et de les faire rectifier, et avec une disposition expresse prévoyant que le contrôle du respect de ces règles est confié à une autorité indépendante (art. 8).

\section{I - LA PROTECTION DES DONNÉES PERSONNELLES DU TRAVAILLEUR}

II convient tout d'abord de souligner l'importante évolution de la législation dans ce domaine, les risques croissants d'actions empiétant sur la vie privée des personnes étant de plus en plus pris en considération. L'essor de cette intervention législative a conduit à une réglementation dotée de ses propres principes et de son propre régime, ce qui permet de reconnaître le droit à la protection des données comme un droit fondamental

3 STC 11/1998 du 13 janvier (BOE 12 février 1998).

4 Loi organique 5/1992 du 29 octobre (BOE 31 octobre 1992) réglementant le traitement automatisé des données à caractère personnel, abrogée et remplacée par la loi 15/1999 du 13 décembre (BOE 14 janvier 2000), elle-même largement abrogée par la récente loi organique 3/2018 du 5 décembre (BOE 6 décembre 2018) sur la protection des données personnelles et la garantie des droits numériques.

5 Directive 95/46 du 24 octobre (JOCE 23 novembre) relative à la protection des personnes physiques à l'égard du traitement des données à caractère personnel et à la libre circulation de ces données.

6 Règlement UE 2016/679 du 27 avril (JOUE 4 mai) relatif à la protection des personnes physiques à l'égard du traitement des données à caractère personnel et à la libre circulation de ces données.

7 Directive UE 2016/680 du 27 avril 2016 (JOUE 4 mai) relative à la protection des personnes physiques à l'égard du traitement des données à caractère personnel par les autorités compétentes. 
bénéficiant d'une autonomie propre. Ceci ne conduit pas seulement à la formation d'un droit qui acquiert sa propre identité et, en tant que tel, une pleine autonomie par rapport à sa référence initiale.

En effet, la protection des données trouve son origine historique dans un renforcement du droit à l'intimité, bien que son extension progressive l'amène à acquérir un contenu propre, qui va au-delà du droit à l'intimité, avec une rare intensité.

Nous sommes encore dans une phase de transition dans la mesure où, bien que la protection des données personnelles couvre des domaines et des contenus plus larges, elle conserve ses liens avec le droit à l'intimité. Ainsi, il n'est pas facile d'expliciter l'affirmation selon laquelle la protection des données va au-delà du droit à l'intimité, tout comme il n'est pas facile de déterminer si cette autonomie conduit à un régime juridique régissant l'exercice et la protection de ce droit différent du régime habituellement appliqué au droit à l'intimité.

Dans ce domaine, les changements technologiques qui se sont généralisés à un rythme inédit au cours des dernières décennies exercent une forte influence, notamment par les processus de numérisation, de traitement de masse de données, de mise en œuvre de l'intelligence artificielle, de mécanismes de vérification de comportements à faible coût grâce à la vérification de la localisation, du son, de l'image, etc.

Toutes ces évolutions technologiques offrent d'énormes possibilités d'obtenir, de stocker et de traiter les données personnelles de tout citoyen - notamment du travailleur - à des fins très diverses. Dans ce contexte, le recours à ces technologies présente des risques considérables d'intrusion dans la sphère privée du citoyen et, plus particulièrement du travailleur. C'est sans aucun doute ce qui a poussé les législateurs européens et nationaux à adopter des mesures visant à protéger la vie privée.

Cependant, même si cette réglementation est motivée par des transformations technologiques, cela ne veut pas dire que sa portée sera limitée au domaine de la technologie, et cela ne constituera pas non plus un facteur permettant de différencier le droit à l'intimité du droit à la protection des données. En effet, la règlementation de référence vise à protéger la sphère privée du travailleur, peu importe que les éventuelles violations surviennent par le biais des technologies numériques ou par des actions plus traditionnelles ne reposant pas sur ce type de technologies.

En revanche, lorsque des mécanismes de protection spécifiques sont prévus pour I'utilisation de certaines méthodes liées au numérique (vidéosurveillance, contrôle du son et de l'image, géolocalisation, traitement informatisé des données, utilisation de réseaux sociaux, contrôles biométriques ou génétiques, etc.) cela concerne à la fois la protection de l'intimité et la protection des données à caractère personnel.

D'un point de vue strictement juridique, la nouveauté la plus notable et largement acceptée concerne l'extension de l'objet de la protection, c'est-à-dire l'extension du contenu et, par conséquent, une intervention qui englobe des renseignements plus divers et plus complets sur la personne; en l'occurrence le salarié. Ainsi, l'attention n'est pas exclusivement portée sur la sphère la plus privée du travailleur, qui est généralement exclue du champ des relations sociales indifférenciées et relève donc de son «intimité ». Elle s'étend également à une autre série d'éléments personnels, plus ou moins publics, que le travailleur entend préserver pour qu'ils n'échappent pas à son contrôle et soient traités indistinctement par quiconque, notamment par l'employeur. II est dès lors possible 
d'affirmer qu'il existe deux sphères, l'une étant le noyau dur de l'intimité et l'autre, plus large, celle de la vie privée.

Cependant, cette extension du domaine de la vie privée n'est pas une simple opération de renforcement de l'objet du droit à l'intimité et résulte de l'identification d'un nouveau droit - la protection des données à caractère personnel - qui va de pair avec l'intimité, mais qui acquiert progressivement sa propre identité. On peut imaginer des cercles concentriques, avec au centre un petit cercle, celui de l'intimité, et autour, un cercle plus grand, celui de la vie privée. En élargissant le champ d'application, on prend également en compte la protection des données personnelles du travailleur qui ne sont pas intimes, bien que cela permette évidemment de protéger celles qui le sont vraiment.

Si l'on admet cette dualité, le premier défi consiste à identifier les aspects qui relèvent de l'intimité et ceux qui relèvent de la vie privée. Cette distinction n'est pas aisée car nul ne peut affirmer qu'il existe des données spécifiques, relevant nécessairement de tel ou tel domaine, en raison du nombre et de l'influence des facteurs:

- caractéristiques de la personne ;

- lieu où elle se trouve ;

- moment des faits ;

- type de relations sociales concernées, ;

- objectif du traitement des données ;

- etc.

Par exemple, l'employeur ne pourra pas exercer le même contrôle sur la vie familiale ou l'activité strictement professionnelle du travailleur pendant sa journée de travail ou en dehors, dans des espaces strictement réservés comme les sanitaires, ou dans les salles de repos de l'entreprise. Le contrôle ne sera pas non plus le même si le travailleur exerce au sein de l'entreprise ou depuis chez lui, si le contrôle concerne la personne du travailleur ou les éléments se trouvant sur son poste de travail, si le contrôle est réalisé en respectant, ou non, les droits fondamentaux classiques (comme les droits d'opinion, d'expression, de réunion, d'association).

La législation a parfois eu recours à la catégorie des données particulièrement sensibles, ce qui peut en partie faire référence à cette différence, mais ne touche pas toujours, ou seulement, cette catégorisation.

En revanche, si la dualité entre intimité et vie privée est acceptée, c'est parce que le régime juridique de protection peut différer. Sans entrer dans les détails, on peut supposer que la différenciation aura des conséquences juridiques, en ce sens que la protection sera plus poussée s'il y a intrusion de la sphère intime que s'il y a simplement intrusion de la sphère privée. Cela pourrait conduire à un régime juridique légèrement différent entre la protection du droit à l'intimité et celle du droit à la vie privée, avec des aspects différents, notamment s'agissant de la propriété, des différents types de consentement de la partie intéressée, de l'établissement éventuel d'une contrepartie économique pour les avantages tirés de leur traitement par l'employeur, de l'exercice du pouvoir de contrôle par les représentants de travailleurs, des règles de procédure relatives à la charge de la preuve de la violation présumée des droits fondamentaux, etc. 


\section{II - LE CHAMP D'APPLICATION SUBJECTIF DE LA PROTECTION : L'EXTENSION À LA PROTECTION DU TRAVAIL INDÉPENDANT}

Dans ce contexte, la tension habituelle entre le travail salarié et le travail indépendant réapparaît, en particulier lorsqu'il se fait via des plateformes numériques, mais aussi plus généralement lorsqu'il concerne des catégories de travailleurs indépendants pour lesquelles les risques de violation de la vie privée sont aussi importants que pour les travailleurs salariés.

Or, il ne semble pas y avoir d'obstacle à ce que la protection de la vie privée et de l'intimité concerne également le travail indépendant : la doctrine de l'efficacité inter privato des droits fondamentaux se présente comme un changement qualitatif de la sphère publique à la sphère privée, pour tous les types de relations juridiques. Dans la mesure où cette construction se fait dans le respect de l'application des droits fondamentaux de tout citoyen, liant non seulement les autorités publiques mais aussi tous les individus, elle pourrait facilement être étendue aux relations juridiques civiles, commerciales ou administratives, à travers lesquelles les indépendants exercent leur activité économique.

À cela s'ajoute le grand avantage de la transversalité des règlements sur la protection des données, qui facilite leur application au domaine du travail indépendant. Ainsi, depuis l'approbation et l'entrée en vigueur du Statut du travail indépendant ${ }^{8}$ en 2007, c'est ce que prévoit aujourd'hui de manière expresse le droit positif lui-même dans le système juridique espagnol. En outre, le texte juridique ne limite pas ces droits aux seuls travailleurs indépendants économiquement dépendants (cas dans lequel la logique protectrice du système juridique est renforcée), mais englobe toutes les formes de travail indépendant.

Toutefois, en pratique, la situation n'est pas aussi simple car la doctrine de l'efficacité inter privato tire son origine des actions d'un employeur dans le domaine du contrat de travail, tant sur le plan juridique que judiciaire.

Au-delà des constructions doctrinales, il semble que la disposition légale n'ait pas su imprégner la réalité sociale, se présentant peut-être comme l'un des exemples paradigmatiques qui illustrent le manque d'efficacité ou d'efficience des réglementations établies à cette fin. Pour le confirmer, il suffit de constater le peu de décisions judiciaires qui ont appliqué la protection des données à caractère personnel au domaine du travail indépendant.

\section{III - LA PROTECTION DES DONNÉES DE L'EMPLOYEUR}

À l'origine, l'efficacité inter privato était basée sur la présence d'un pouvoir privé fort, ayant la capacité de nuire aux droits fondamentaux, dans le domaine du travail. De ce fait, le travailleur a toujours été considéré comme la victime de ces dommages et l'employeur comme le responsable. À cela s'ajoute l'idée que les droits fondamentaux étaient réservés aux citoyens en tant que personnes physiques, et non aux personnes morales, l'employeur s'en trouvant donc dépourvu. Cependant, la garantie des droits fondamentaux ayant beaucoup évolué, le scénario commence à être différent.

8 Loi 20/2007 du 11 juillet 2007 (BOE 12 juillet 2007). 
Tout d'abord, l'employeur est non seulement une personne morale mais peut également être une personne physique. En outre, au fil du temps, le catalogue des droits fondamentaux intègre certains droits détenus de manière collective, et donc par une personne juridique particulière, comme par exemple la liberté syndicale et le droit de grève dans son aspect collectif.

Dès lors, il est difficile de dissocier cette reconnaissance accordée au syndicat en tant qu'organisation du reste des personnes morales. II est évident que certains droits fondamentaux ne peuvent être attribués qu'aux personnes physiques : I'habeas corpus, le droit à la vie et à l'intégrité physique, le droit à l'éducation, etc.

Toutefois, il convient également de souligner que d'autres droits fondamentaux pourraient s'appliquer aux personnes physiques et morales, notamment ceux relatifs à la protection des données à caractère personnel. La preuve en est que la Cour constitutionnelle, qui avait initialement déclaré que les droits fondamentaux ne pouvaient être attribués qu'à des personnes physiques, a finalement admis, avec certaines nuances toutefois, que les personnes morales peuvent également être titulaires de certains droits fondamentaux.

L'une des conséquences les plus immédiates de la diffusion mondiale des technologies de l'information et de la communication est l'effet multiplicateur exponentiel des big data sur le droit à l'intimité et à la vie privée.

En outre, avec ce type de processus technologiques, l'importance du patrimoine matériel de l'entreprise diminue lorsqu'on le compare au capital total de l'entreprise, l'image commerciale de l'entreprise acquérant une importance particulière, de même que sa réputation dans le domaine d'activité économique, notamment dans ses relations avec les investisseurs, les clients et les fournisseurs. Il convient donc de se demander s'il est possible de faire valoir le droit à l'image, y compris - à certaines fins - le droit à l'honneur de l'employeur du moins en sa qualité de personne physique.

Une problématique se pose néanmoins : le fait d'attribuer de manière subjective ces droits à davantage d'entités, notamment à l'employeur, ne saurait se traduire par un enrichissement mutuel et un approfondissement général de l'exercice des droits correspondants et, par conséquent, par une protection étendue des intérêts juridiques en jeu, sous la forme d'une opération gagnant-gagnant. Bien souvent au contraire, lorsque l'employeur exerce et jouit de ce droit, cela entraîne indirectement à l'égard de l'autre partie (le travailleur) la suppression, ou du moins la restriction, du même droit fondamental ou d'un droit différent qui s'en trouve affecté dans la pratique.

Ainsi, de manière générale, la sphère privée de l'employeur peut affecter le principe général de transparence qui est aujourd'hui un instrument clé pour assurer la jouissance d'autres droits, en particulier des droits fondamentaux. Par exemple, le fait que l'employeur en tant que personne morale puisse invoquer la défense de sa vie privée pour conditionner ou limiter les droits à l'information des représentants de travailleurs. Ainsi, ce n'est pas par hasard que la récente loi sur les secrets commerciaux exclut expressément du champ protégé, au profit des entreprises, l'exercice du droit des travailleurs à être informés et consultés, l'obtention par les représentants syndicaux des informations constituant le secret commercial étant ainsi considérée comme licite ${ }^{9}$ (art. 2.1.C). 
De même, le droit à l'image et, par extension, le droit à la réputation d'une entreprise, peuvent être particulièrement affectés aujourd'hui par l'impact que l'image ou la réputation ont sur les réseaux sociaux, lesquelles peuvent donc être fortement atteintes selon l'usage que les salariés font de ces réseaux sociaux. II ne faut pas non plus oublier que désormais, l'image de l'entreprise n'est pas seulement véhiculée par des éléments matériels (logos, slogans) ou des messages publicitaires, mais aussi et surtout par les actions des salariés et leur manière de s'exprimer au sujet de leur entreprise. Dans ce domaine, la Cour constitutionnelle, comme la Cour de Justice de l'Union Européenne et la Cour Européenne des Droits de l'Homme, invoquent dans les textes correspondants la garantie de la liberté d'entreprise, notamment la défense de la productivité, en tant que droits « fondamentaux» reconnus aux employeurs, afin d'admettre des limitations ou des restrictions importantes dans l'exercice et la jouissance des droits fondamentaux correspondants des travailleurs. À cet égard, on se contentera de mentionner les restrictions considérables que la jurisprudence constitutionnelle a imposées, à maintes reprises, à la liberté d'expression des travailleurs, par rapport à celle attribuée aux citoyens en général, découlant de la garantie du bon fonctionnement de la liberté d'entreprise.

Toutefois, la protection des droits et des intérêts attribués aux travailleurs et aux employeurs dans le domaine de la protection des données à caractère personnel ne saurait être assurée de manière paritaire, et ce pour plusieurs raisons :

- Premièrement, la reconnaissance de la liberté d'entreprise et la protection de la productivité des entreprises ne figurent pas dans le catalogue officiel des droits fondamentaux et des libertés publiques au sens strict de la Constitution espagnole.

- Deuxièmement, il ne faut pas oublier que le déséquilibre contractuel des relations privées a joué un rôle décisif dans la reconnaissance de l'efficacité inter privato, ce qui aurait conduit à accorder une plus grande importance à la protection des droits fondamentaux de la partie contractante faible, en particulier dans le domaine du travail.

- Troisièmement, bien qu'il soit fait parfois référence à des droits identiques, les droits dont jouissent l'employeur et le travailleur diffèrent clairement en termes de fonctionnalité et de finalité ; du côté des travailleurs, la jouissance de ces droits est liée à la protection de leur dignité en tant que personne physique, alors que du côté de l'employeur, elle est simplement liée à l'intérêt juridique des bénéfices de l'entreprise, qui mérite certes d'être protégé, mais qui a moins de valeur que la finalité précédente liée à la dignité.

Pour résumer, la différenciation entre la protection de l'intimité et la protection de la vie privée en tant que droits fondamentaux à part entière peut amener à considérer la première comme étroitement liée à la dignité de la personne, et la seconde comme beaucoup plus éloignée de celle-ci.

En outre, la protection des valeurs d'ordre public, qui vont au-delà de la protection des intérêts des travailleurs et des employeurs en tant que personnes physiques, figure parfois dans la protection des intérêts publics. Par exemple, si un salarié est licencié ou sanctionné au motif qu'il a dénoncé son employeur pour des comportements susceptibles d'entraîner des attaques dans le domaine de la cybersécurité, cette sanction est déclarée nulle et non avenue, ce qui constitue une expression supplémentaire de la garantie d'indemnisation ${ }^{10}$.

10 Art. 20.2 du décret-loi royal 12/2018 du 7 septembre 2018 sur la sécurité des réseaux et des systèmes d'information (BOE 8 septembre 2018). 
Enfin, le fait de reconnaître au travailleur la possibilité d'enregistrer des informations aux fins de son éventuelle défense dans le cadre d'un procès, avec une atteinte plus grande à la vie privée de l'employeur que celle qui lui serait accordée s'il venait à porter atteinte à la vie privée du travailleur pour les mêmes raisons. Citons l'exemple du travailleur qui enregistre secrètement une conversation avec son employeur en pensant qu'il va lui communiquer les motifs sous-jacents de son licenciement ${ }^{11}$.

\section{IV - LES LIMITES DU CONSENTEMENT PERSONNEL}

En dehors du cadre du travail, la notion d'habeas data se consolide. Ceci implique l'attribution de la propriété des données à caractère personnel au citoyen concerné par les informations correspondantes, qui peut accepter ou non que ces données soient collectées et traitées par des tiers. Dans cette optique, on attribue souvent une valeur décisive au consentement de la personne concernée à l'utilisation de ces données. Cependant, dans le domaine du travail, certaines spécificités nécessitent de nuancer à la fois la règle de l'habeas data et le rôle qui doit être attribué au consentement de la personne considérée.

Dans le cadre de ses devoirs légaux et de ses obligations contractuelles, l'employeur est parfois tenu de disposer et de traiter toute une série de données à caractère personnel concernant le travailleur.

En voici quelques exemples:

- afin de respecter les obligations d'affiliation, d'enregistrement et de cotisation à la sécurité sociale pour le travailleur, il est nécessaire de connaître certaines données personnelles ;

- pour le paiement du salaire, il peut être nécessaire de disposer des coordonnées bancaires personnelles du travailleur ;

- pour appliquer certaines mesures de promotion de l'emploi, l'employeur doit connaître certaines données personnelles du salarié ;

- afin de conclure certains accords contractuels avec un salarié particulier, l'employeur doit savoir si le salarié remplit certaines exigences déterminantes pour son recrutement ;

- pour remplir ses obligations dans le domaine de la prévention des risques professionnels, l'employeur doit disposer de certaines informations sur la situation personnelle du travailleur ;

- pour pouvoir donner certaines instructions au travailleur, il doit connaître son profil personnel,

- etc.

Par ailleurs, pour pouvoir développer efficacement son pouvoir de contrôle, l'employeur doit avoir accès aux données personnelles du travailleur, même si de nombreuses limites sont fixées pour garantir la protection de la vie privée du travailleur.

Cependant, il existe d'autres données qui, bien qu'utiles et bénéfiques pour améliorer la gestion de l'entreprise, ne sont ni nécessaires à l'accomplissement de certaines obligations légales, ni indispensables à l'exercice du pouvoir de gestion et de contrôle de l'entreprise.

11 STS, civil, 20 novembre 2014, rec. 3402/2012, ECLI:ES:TS:2014:5215. 
Il ne s'agit donc pas ici de contester le fait que le travailleur bénéficie également du droit à la protection de ses données à caractère personnel dans le cadre de l'exercice de ses fonctions, car nul ne peut remettre en cause le fait que le droit à la vie privée en tant que droit fondamental a également un effet inter privatos.

Néanmoins, il convient de souligner que le principe de l'habeas data ne constitue pas un critère général pour délimiter l'espace de vie privée du travailleur dans le domaine des relations de travail et, par conséquent, son espace de protection.

En lien avec ce qui précède, la gestion des algorithmes apparaît de plus en plus importante à l'ère numérique. Cette gestion est essentielle, non seulement pour éviter d'empiéter sur la vie privée, mais aussi pour éviter que, dans le contexte de l'intelligence artificielle, cette gestion n'entraîne des effets biaisés qui ont des conséquences néfastes sur d'autres droits fondamentaux, à commencer par les risques de traitements discriminatoires découlant de ces algorithmes.

Par ailleurs, le consentement à l'utilisation des données à caractère personnel, qui ouvre pratiquement toutes les portes pour leur traitement dans les domaines extra-professionnels, perd beaucoup de sa valeur lorsqu'il s'agit d'un emploi salarié ou, plus généralement, d'une activité professionnelle salariée ou indépendante où l'on constate un déséquilibre contractuel évident entre les parties. Le consentement donné par le travailleur à son employeur pour l'utilisation de ses données à caractère personnel n'a que très peu de valeur lorsqu'on sait que, dans la plupart des cas, le travailleur n'est pas libre.

C'est pourquoi le principe général de la non-renonciation aux droits fondamentaux revêt un caractère plus fort dans le domaine de l'emploi, à tel point que le fait que le travailleur consente ou non au traitement de ses données à caractère personnel, est considéré comme sans importance. Dans le domaine des relations de travail, il revient donc à la réglementation de l'État - ou à la convention collective - de consentir ou d'interdire l'utilisation de données à caractère personnel à des fins justifiées ou non pour l'exercice de fonctions et de pouvoirs commerciaux, avec une reconnaissance marginale du consentement individuel du travailleur.

Dans le domaine de l'emploi, au-delà du consentement individuel, il devrait être primordial d'imposer trois éléments fondamentaux :

- en premier lieu, une obligation de transparence et d'information pour expliquer à chacun que leur données seront traitées et à quelles fins elles seront utilisées ;

- ensuite, un lien strict entre le traitement des données et la finalité spécifique pour laquelle le consentement individuel est autorisé, sans possibilité d'autres utilisations et avec la destruction des données une fois que la finalité est atteinte ou caduque ;

- enfin, un contrôle approfondi par les représentants de travailleurs concernant l'utilisation et le traitement des données par l'employeur. Le processus de traitement numérique doit être régi par le principe de transparence et, par conséquent, permettre aux salariés et à leurs représentants de contrôler la bonne utilisation des données.

Enfin, il arrive que l'habeas data, le consentement du propriétaire et le profitéconomique que l'entreprise tire du traitement des données à caractère personnel, soient liés. Certains défendent donc le droit que la personne titulaire soit indemnisée financièrement pour 
I'utilisation de ses données à caractère personnel. Cette question se pose d'autant plus avec les données fournies aux entreprises concernées par les utilisateurs et consommateurs.

Toutefois, il faut nuancer lorsque l'on transpose ce nouvel aspect de la compensation financière au domaine du travail dans la mesure où il est évidemment inapproprié d'exiger de l'employeur une telle compensation financière quand le traitement des données répond à un devoir légal ou une obligation contractuelle. Cela ne semble pas non plus raisonnable lorsque l'accès aux données à caractère personnel est indispensable à l'exercice du pouvoir de gestion et de contrôle auquel l'employeur a droit, en vertu de la loi.

D'autre part, dans le domaine de la garantie des droits fondamentaux, le consentement, assorti d'une compensation financière, n'élimine pas complètement les réserves et la méfiance découlant de la position de supériorité de l'employeur sur le travailleur. Dans ce cas, la «non-renonciation » aux droits doit impliquer également l'« indisponibilité » des droits, en ce sens que l'obtention d'une contrepartie financière pour avoir donné son consentement ne doit pas nécessairement conduire à un transfert de données licite de manière générale. La monétisation de la non-jouissance des droits fondamentaux est essentielle à cet égard, du moins en ce qui concerne le noyau dur de ces droits, qui doit être protégé de manière inconditionnelle.

II serait alors possible de trouver ici une autre distinction entre intimité et vie privée : les données relatives à l'intimité ne doivent en aucun cas être disponibles, ni même en échange d'une compensation financière au titre du profit économique obtenu par l'employeur pour sa gestion et son traitement, au point que cela devait être compris comme un principe directeur dans le domaine du travail. À l'inverse, le transfert de données à caractère personnel non liées à l'intimité et faisant l'objet d'une compensation financière ne doit pas soulever d'objections ni d'empêchements outre mesure.

\section{V - L'EXERCICE DU POUVOIR DE CONTRÔLE}

La plus grande nouveauté dans le domaine de la protection des données personnelles dans le domaine du travail concerne tout ce qui touche à l'exercice du pouvoir de contrôle de l'employeur. Étant donné que le traitement des données ne peut entraîner une violation des droits fondamentaux, il existe certaines données dont le contrôle est exclu quelle que soit la finalité : les données personnelles révélant l'origine ethnique ou raciale, les opinions politiques, les croyances religieuses ou philosophiques, l'appartenance à un syndicat, la vie sexuelle, l'orientation sexuelle, ainsi que toute autre donnée qui pourrait être interprétée comme portant atteinte aux droits fondamentaux et aux libertés publiques ${ }^{12}$.

D'autres données peuvent être contrôlées, sous réserve du consentement du travailleur, ou se révéler nécessaires au respect des obligations et à l'exercice des droits incombant à l'employeur: santé du travailleur, données génétiques et biométriques visant à identifier sans équivoque une personne physique.

Outre ce qui précède, il est établi que, pour éviter des situations discriminatoires, le seul consentement du travailleur ne suffit pas à lever l'interdiction de traitement des données dont le but principal est d'identifier son idéologie, son appartenance à un syndicat, sa religion, son orientation sexuelle, ses croyances ou son origine raciale ou ethnique.

12 Art. 9 du règlement UE 2016/679. 


\section{A - LES DISPOSITIFS NUMÉRIQUES À LA DISPOSITION DU TRAVAILLEUR}

En ce qui concerne la possibilité de contrôle de l'employeur grâce à l'accès aux dispositifs numériques (ordinateurs portables, tablettes numériques, téléphones mobiles et similaires), les travailleurs ont le droit à la protection de leur intimité lorsqu'ils utilisent des dispositifs numériques mis à disposition par leur employeur (article 20 bis ET). L'employeur peut accéder au contenu issu de l'utilisation des médias numériques fournis aux salariés dans le seul but de contrôler le respect des obligations professionnelles et de garantir l'intégrité de ces dispositifs ${ }^{13}$.

En ce qui concerne le recours aux communications électroniques par le salarié, la Cour constitutionnelle prend tout d'abord en considération le fait que cela peut affecter la confidentialité des communications (article 18.3 CE) et le droit à l'intimité (article 18.1 CE), de sorte que ce qui est stocké dans l'ordinateur personnel du salarié est concerné par ces droits. Toutefois, elle a considéré comme recevables l'organisation et la réglementation de I'utilisation par le travailleur des moyens informatiques appartenant à l'entreprise, ainsi que la capacité de cette dernière à superviser et à contrôler le respect des obligations relatives à I'utilisation des ressources en question, toujours dans le respect des droits fondamentaux ${ }^{14}$.

\section{B - VIDÉOSURVEILLANCE ET ENREGISTREMENT AUDIO}

La loi prévoit que l'employeur peut traiter les images obtenues par des systèmes de caméras ou de caméras vidéo dans le cadre de l'exercice de ses fonctions de contrôle des travailleurs, à condition que ces fonctions soient exercées conformément au cadre juridique et dans les limites inhérentes à celui-ci. En aucun cas, des systèmes d'enregistrement audio ou de vidéosurveillance ne peuvent être installés dans des lieux destinés au repos ou aux loisirs des travailleurs, tels que les vestiaires, les toilettes, les cantines et autres. L'utilisation cachée de systèmes de vidéosurveillance n'est autorisée que lorsque les risques liés à la sécurité des installations, des biens et des personnes découlant de l'activité exercée sur le lieu de travail sont réels et toujours dans le respect du principe de proportionnalité, du principe d'intervention minimale et des garanties prévues pour les dispositifs de vidéosurveillance ${ }^{15}$. La règle générale veut que le travailleur soit informé de leur installation.

13 Art. 87 de la loi organique 3/2018 du 5 décembre (BOE 6 décembre 2018) sur la protection des données à caractère personnel et la garantie des droits numériques.

14 Selon la loi, l'entreprise peut accéder à une conversation électronique entre deux travailleurs par l'intermédiaire d'un programme de messagerie qu'ils ont eux-mêmes installé sur un ordinateur destiné à l'usage commun de tous les travailleurs et qui n'est pas protégé par un mot de passe (STC 241/2012 du 17 décembre). Lorsque la convention collective interdit l'utilisation de la messagerie électronique en dehors du cadre du travail, l'entreprise est autorisée à contrôler son utilisation, afin de vérifier que le travailleur a respecté ses obligations et devoirs professionnels (STC 170/2013, 7 octobre). Toutefois, la Cour suprême considère que cette règle est limitée à la sphère de la juridiction sociale, sans s'étendre aux poursuites pénales (STS, pénal, 16 juin 2014 , rec. 2229/2013, ECLI:ES:TS:2014:2844).

15 Art. 89 de la loi organique 3/2018 du 5 décembre 2018 (BOE 6 décembre) sur la protection des données à caractère personnel et la garantie des droits numériques. Dans une entreprise de jeux, il n'est pas indispensable pour la sécurité et le bon fonctionnement de l'entreprise d'installer des équipements pour enregistrer les conversations entre les salariés et les clients, lorsqu'un système de capture d'images à distance est déjà en place (STC 98/2000 du 10 avril). L'installation par l'entreprise d'un système de télévision en circuit fermé pour surveiller certains postes de travail où l'argent de l'entreprise est manipulé est justifiée et proportionnée (STC 186/2000 du 10 juillet). 
Toutefois, en appliquant le principe de proportionnalité dans des situations particulières, il est permis de dissimuler la surveillance, en particulier lorsqu'il existe des soupçons fondés de manquement grave de la part du travailleur, que ces soupçons sont significatifs au regard des dommages causés au patrimoine de l'entreprise et qu'il a été prouvé qu'il n'existait pas d'autres moyens moins intrusifs de confirmer les manquements du travailleur ${ }^{16}$.

\section{C - SYSTÈmES DE GÉOlOCALISATION}

La surveillance du travailleur en dehors de l'enceinte de l'entreprise est autorisée lorsqu'il s'agit de vérifier que celui-ci s'acquitte de ses obligations professionnelles en dehors des locaux de l'entreprise. L'employeur peut recourir à la géolocalisation et traiter les données obtenues dans le cadre de l'exercice de ses fonctions de contrôle des salariés, à condition que ces fonctions soient exercées conformément au cadre juridique et dans les limites inhérentes à celui-ci.

La mise en place de systèmes de géolocalisation doit être connue du travailleur, et l'entreprise doit l'informer de la finalité du traitement des données ainsi obtenues, faute de quoi l'employeur peut être sanctionné pour violation de la réglementation sur la protection des données à caractère personnel.

\section{D - DONNÉES BIOMÉTRIQUES}

Obtenues grâce à un procédé technique spécifique, les données biométriques sont les caractéristiques physiques, physiologiques ou comportementales d'une personne physique, qui permettent ou confirment l'identification unique de cette personne, comme les photos de visage ou les données dactyloscopiques.

Les données biométriques comprennent notamment les empreintes digitales, les modèles rétiniens, la forme du visage, la voix, la forme de la main, le réseau veineux, la calligraphie ou toute autre donnée similaire permettant l'identification unique d'une personne. Ces données peuvent être utilisées pour contrôler la présence des travailleurs sur le lieu de travail ou les horaires. Dans ces cas, la réglementation exige que le travailleur donne son consentement explicite. En outre, le travailleur est tenu d'être dûment informé de la finalité pour laquelle ces données sont collectées.

\section{E - CONTRÔLE DES DONNÉES GÉNÉTIQUES}

Les données génétiques désignent les caractéristiques génétiques héritées ou acquises d'une personne physique qui fournissent des informations uniques sur sa physiologie ou sa santé, obtenues notamment par l'analyse d'un échantillon biologique de cette personne. Il s'agit d'une analyse chromosomique de l'ADN, de l'ARN ou de tout autre élément qui fournit des informations équivalentes. Dans ces cas, leur utilité est très liée à l'état de santé du travailleur, dans la mesure où ces données permettent de détecter des maladies ou autres problèmes de santé qui constituent un obstacle au travail du salarié.

Ce processus peut toutefois détecter de nombreuses autres données personnelles qui n'ont aucune pertinence sur le plan professionnel et viennent par conséquent empiéter beaucoup plus sur la vie privée du travailleur. Par conséquent, ces données ne sont autorisées que si ce qu'elles cherchent à renseigner ne peut être obtenu autrement. Par

16 CEDH, Grande Chambre, 17 octobre 2019, affaire López Ribalda II. 


\section{Protection des donnéEs Personnelles en Espagne}

ailleurs, même dans les cas où elles sont licites, elles sont soumises aux mêmes exigences que les données biométriques : consentement du travailleur et information du travailleur sur la finalité de leur traitement.

\section{F - CONTRÔLE PENDANT LA JOURNÉE ET DÉCONNEXION}

Le pouvoir de contrôle doit en principe être exercé pendant la journée de travail. II peut toutefois être exercé en dehors, à titre exceptionnel. Pour cela, il existe des règles précises sur le temps de travail et les périodes de repos, qui déterminent indirectement l'étendue du pouvoir de contrôle de l'employeur.

Dans ce contexte, il est particulièrement important de reconnaître le droit du travailleur à la déconnexion, ce qui implique notamment l'obligation de respecter la vie privée du travailleur pendant la période de déconnexion et, par conséquent, une limitation objective du pouvoir de contrôle de l'employeur.

\section{JESÚS CRUZ VILLALÓN}

Professeur de Droit du travail et de la Sécurité sociale, Université de Séville.

Thèmes de recherche : Les droits fondamentaux face aux changements du travail dans la nouvelle économie, droit comparé de la négociation collective en Europe, politiques salariales: du salaire minimum à la négociation collective.

\section{Publications:}

$\sim$ J. Cruz Villalón, " Las facultades de control del empleador ante los cambios organizativos y tecnológicos ", Temas Laborales, $n^{\circ}$ 150, 2019.

$\sim$ J. Cruz Villalón, " La jurisprudencia del Tribunal Europeo de Derechos Humanos en materia laboral », Temas Laborales, n 145, 2018. 